Research Article

\title{
Reanalysis of Modified Structures by Adding or Removing Substructures
}

\author{
Yong-Su Kim ${ }^{1}$ and Hee-Chang Eun $\mathbb{D}^{2}$ \\ ${ }^{1}$ Department of Architectural Engineering, Chung-Ang University, Seoul, Republic of Korea \\ ${ }^{2}$ Department of Architectural Engineering, Kangwon National University, Chuncheon, Republic of Korea
}

Correspondence should be addressed to Hee-Chang Eun; heechang@kangwon.ac.kr

Received 22 June 2017; Accepted 3 October 2017; Published 8 April 2018

Academic Editor: Gianmarco de Felice

Copyright ( $) 2018$ Yong-Su Kim and Hee-Chang Eun. This is an open access article distributed under the Creative Commons Attribution License, which permits unrestricted use, distribution, and reproduction in any medium, provided the original work is properly cited.

\begin{abstract}
This study considers structural reanalysis owing to the modification of structural elements including (1) addition of substructures, (2) removal of substructures, and (3) changes in design variables. Coupling and decoupling reanalysis methods proposed in the study are performed by using the concept of compatibility conditions at interface nodes between the substructures or between the original structure and the substructures. Subsequently, a generalized inverse method to describe constrained responses is modified to obtain the reanalysis responses. In this study, constrained equilibrium equations are modified to consider a reanalysis of a structure with the addition and removal of statically stable or unstable substructures. The proposed reanalysis method is examined by using five examples of handling coupling and decoupling reanalysis of a truss structure.
\end{abstract}

\section{Introduction}

The design stage of complex structures may include the addition of substructures with respect to the initial structure or the removal of substructures from the original structure; this requires further analysis. Structural reanalysis involves predicting structural responses of a modified structure owing to changes in structural members by using initial information without repeatedly solving a complete set of modified simultaneous equations.

The reanalysis method is categorized into direct methods (exact methods) and iterative methods (approximate methods). A direct approach is efficient given changes in a few structural members. In contrast, an approximate approach is suitable given changes in several members. Exact approaches were first proposed in studies by Sherman and Morrison [1] and Woodbury [2]. Nair [3] mentioned that various reanalysis methods were only valid for small perturbations in structural parameters. The aforementioned studies considered large perturbations in structural parameters. Hager [4] reviewed the formulae proposed by Sherman-Morrison and Woodbury that relate the inverse of a matrix after a small-rank perturbation to the inverse of the original matrix. Akgun et al.
[5] extended low-cost linear reanalysis in the spirit of the Sherman-Morrison and Woodbury formulae to update the inverse of a matrix.

Kirsch and Liu $[6,7]$ provided first-order reduced basis expressions for exact displacements and stresses in terms of cross-sectional variables. The concept of structural rigid body motion eigenvectors and a generalized structural compliance matrix was adopted by Huang and Verchery [8] to consider an exact structural static reanalysis method for locally modified structures. Cheng et al. [9] considered a structural static reanalysis due to added DOFs (degrees of freedom) by using a Guyan condensation method and an extrapolation method.

Liu et al. [10] presented a direct reanalysis method with added supports. Liu et al. [11] proposed a static reanalysis method given the modification of supports by using modified master stiffness matrices, a rank-one decomposition of the corresponding incremental stiffness matrix, and a sparse Cholesky rank-one update/downdate algorithm. Liu and Yue [12] considered static reanalysis problem with modification of deleting some supports using the initial information and preserving the ease of implementation. The virtual distortion method (VDM) has been extensively developed and proved to 
be a versatile reanalysis tool in various applications, including structures and truss-like systems. Kolakowski et al. [13] demonstrated the capabilities of the VDM both in statics and dynamics. Liu et al. [14] provided a superelement-based VDM to improve the efficiency of the FEM updating of large-scaled bridges by using static information. Garcia de Jalon and Viadero [15] provided a linear static reanalysis method of structures based on the displacement method with respect to the addition, elimination, or substructuring of one or more elements. Cheikh and Coorevits [16] presented a direct method to introduce the concept of a reflexive inverse and a decomposition method for a stiffness matrix to avoid its inversion.

Three possible modifications of substructures are considered as follows [7]:

(1) Case in which the number of DOFs is reduced due to deletion of members and joints

(2) Case in which the number of DOFs is increased due to addition of members and joints

(3) Case in which the numerical values of variables are modified and the number of DOFs is unchanged

The dimensions of a stiffness matrix can increase or decrease corresponding to the addition or deletion of substructures, respectively. The addition and deletion of substructures are considered as the restriction and release, respectively, of a constrained condition with respect to existing responses. Thus, reanalysis commences with a concept of describing constrained responses. The constrained response with respect to the satisfying constraints uses a generalized inverse method [17] that is explicitly calculated. Response variation along the constrained path that deviates from the initial response path is estimated by constraint forces such that it is executed on the initial structure. Response variation of structural members is estimated to predict the response of the remaining members by using the Guyan condensation method.

This study considers direct reanalysis methods to predict modified responses on the above three modification cases in a truss structure. The basic concept in the study originated from the extension of constraints of the compatibility conditions at free interfaces between structures and substructures. The study derives modification forms of a constrained equilibrium equation by using a generalized inverse method to describe the resulting responses due to the addition and deletion of stable or unstable substructures. Five different case studies of coupling and decoupling of substructures in a truss structure are considered, and the validity of the proposed methods is illustrated.

\section{Formulation}

2.1. Generalized Inverse Method. The reanalysis of an original structure due to the attachment or removal of substructures is performed by using constraints. Reanalysis is performed by restricting or releasing constraint conditions by attaching or removing structural members. The generalized inverse method for constrained responses utilized in the study is summarized in the following section.

The equilibrium equation of $n$ DOF original structure is expressed as follows:

$$
\mathbf{K}_{0} \mathbf{u}_{0}=\mathbf{F} \text {, }
$$

where $\mathbf{K}_{0}$ and $\mathbf{u}_{0}$ denote the $n \times n$ symmetric and positivedefinite stiffness matrix and $n \times 1$ displacement vector at the original state, respectively, and $\mathbf{F}$ corresponds to the $n \times 1$ load vector.

With respect to the process of structural synthesis, it is necessary to consider compatibility conditions at interface nodes between the original structure and the substructures as constraints. It is assumed that the structural response is subjected to $m(n>m)$ linear constraints as follows:

$$
\mathbf{A u}=\mathbf{b},
$$

where $\mathbf{A}$ denotes an $m \times n$ coefficient matrix, u denotes an $n \times 1$ constrained displacement vector, and $\mathbf{b}$ denotes an $m \times 1$ vector. All elements of the vector $\mathbf{b}$ should correspond to zeros when compatibility conditions at interface nodes in synthesizing substructures are considered. The basic property of the Moore-Penrose inverse and the least square method is used, and (1) and (2) are combined to derive a resulting constrained equilibrium equation as follows:

$$
\mathbf{u}=\mathbf{u}_{0}+\mathbf{K}_{0}^{-1 / 2}\left(\mathbf{A} \mathbf{K}_{0}^{-1 / 2}\right)^{+}\left(\mathbf{b}-\mathbf{A} \mathbf{u}_{0}\right),
$$

where “+” denotes the Moore-Penrose inverse and $\mathbf{u}_{0}=\mathbf{K}_{0}^{-1} \mathbf{F}$.

The second term in the right-hand side of (3) denotes the variation in the displacement vector due to the existence of constraints. Both sides of (3) are premultiplied by $\mathbf{K}_{0}$, and thus the second term in the right-hand side of the resulting equation corresponds to the constraint forces required to satisfy the constraints. The constraint forces $\mathbf{F}^{c}$ are expressed as follows:

$$
\mathbf{F}^{\mathcal{C}}=\mathbf{K}_{0}^{1 / 2}\left(\mathrm{AK}_{0}^{-1 / 2}\right)^{+}\left(\mathbf{b}-\mathbf{A} \mathbf{u}_{0}\right) .
$$

Equation (3) is modified to describe the constrained response for establishing structural reanalysis, and reanalysis methods of several types of modified structures are thereby provided. The study presents coupling and decoupling methods to describe resultant responses due to the attachment and removal of substructures, respectively.

2.2. Attachment of Substructures. It is necessary for a static response to be continuous at the end nodes of a finite element model for structural members. It indicates that the compatibility conditions at interface nodes between the adjacent members should be satisfied. Structural synthesis is an analytical process to assemble substructures as shown in Figure 1(a). Substructures are assembled into the entire structure as shown in Figure 1(b) by using a compatibility condition. The assembled substructures correspond to stable substructures of a full-ranked stiffness matrix or the floating substructures of a rank-deficient stiffness matrix.

2.2.1. Addition of Stable Substructures. The original structure and substructures are modeled by using finite elements. 


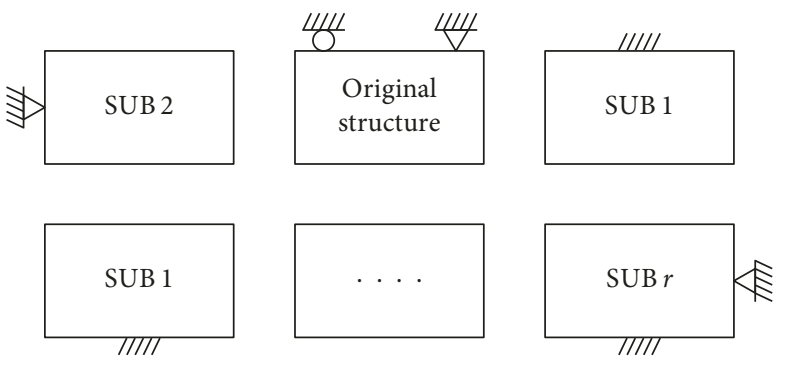

(a)

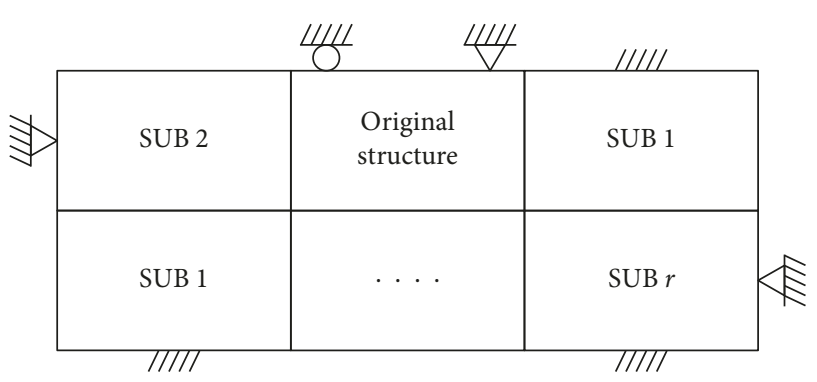

(b)

FIGURE 1: Synthesis of an original structure and $r$ substructures: (a) separated substructures; (b) synthesized substructures.

It is necessary for the static response due to the addition of substructures to be continuous at the interface DOFs. Reanalysis involves predicting displacement variation caused by interactive forces.

The original structure and $r$ statically stable substructures shown in Figure 1 are synthesized to divide the original structure and each substructure into internal elements and interface elements including boundary DOFs as follows:

$$
\begin{aligned}
& {\left[\begin{array}{ll}
\mathbf{K}_{a a}^{(o)} & \mathbf{K}_{a b}^{(o)} \\
\mathbf{K}_{b a}^{(o)} & \mathbf{K}_{b b}^{(o)}
\end{array}\right]\left[\begin{array}{c}
\mathbf{u}_{a}^{(o)} \\
\mathbf{u}_{b}^{(o)}
\end{array}\right]=\left[\begin{array}{c}
\mathbf{F}_{a}^{(o)} \\
\mathbf{F}_{b}^{(o)}
\end{array}\right],} \\
& {\left[\begin{array}{cc}
\mathbf{K}_{a a}^{(1)} & \mathbf{K}_{a b}^{(1)} \\
\mathbf{K}_{b a}^{(1)} & \mathbf{K}_{b b}^{(1)}
\end{array}\right]\left[\begin{array}{c}
\mathbf{u}_{a}^{(1)} \\
\mathbf{u}_{b}^{(1)}
\end{array}\right]=\left[\begin{array}{c}
\mathbf{F}_{a}^{(1)} \\
\mathbf{F}_{b}^{(1)}
\end{array}\right], \ldots,} \\
& {\left[\begin{array}{ll}
\mathbf{K}_{a a}^{(l)} & \mathbf{K}_{a b}^{(l)} \\
\mathbf{K}_{b a}^{(l)} & \mathbf{K}_{b b}^{(l)}
\end{array}\right]\left[\begin{array}{c}
\mathbf{u}_{a}^{(l)} \\
\mathbf{u}_{b}^{(l)}
\end{array}\right]=\left[\begin{array}{c}
\mathbf{F}_{a}^{(l)} \\
\mathbf{F}_{b}^{(l)}
\end{array}\right], \ldots,} \\
& {\left[\begin{array}{ll}
\mathbf{K}_{a a}^{(r)} & \mathbf{K}_{a b}^{(r)} \\
\mathbf{K}_{b a}^{(r)} & \mathbf{K}_{b b}^{(r)}
\end{array}\right]\left[\begin{array}{c}
\mathbf{u}_{a}^{(r)} \\
\mathbf{u}_{b}^{(r)}
\end{array}\right]=\left[\begin{array}{c}
\mathbf{F}_{a}^{(r)} \\
\mathbf{F}_{b}^{(r)}
\end{array}\right],}
\end{aligned}
$$

where " $a$ " and " $b$ " denote the internal and interface elements, respectively. The superscript " $o$ " indicates the original structure, and " $l$ " denotes the $l$ th added substructure. The substructures are attached at interface nodes of the original structure.

The equilibrium equation is condensed by using the Guyan method to reduce the computational operation. Displacements corresponding to internal DOFs from the first equation at each simultaneous equilibrium equation of (5) are derived as follows:

$$
\begin{aligned}
\mathbf{u}_{a}^{(o)} & =\mathbf{K}_{a a}^{(o)^{-1}}\left(\mathbf{F}_{a}^{(o)}-\mathbf{K}_{a b}^{(o)} \mathbf{u}_{b}^{(o)}\right), \\
\mathbf{u}_{a}^{(1)} & =\mathbf{K}_{a a}^{(1)^{-1}}\left(\mathbf{F}_{a}^{(1)}-\mathbf{K}_{a b}^{(1)} \mathbf{u}_{b}^{(1)}\right), \ldots, \\
\mathbf{u}_{a}^{(l)} & =\mathbf{K}_{a a}^{(l)^{-1}}\left(\mathbf{F}_{a}^{(l)}-\mathbf{K}_{a b}^{(l)} \mathbf{u}_{b}^{(l)}\right), \ldots, \\
\mathbf{u}_{a}^{(r)} & =\mathbf{K}_{a a}^{(r)^{-1}}\left(\mathbf{F}_{a}^{(r)}-\mathbf{K}_{a b}^{(r)} \mathbf{u}_{b}^{(r)}\right),
\end{aligned}
$$

where the superscript "- 1 " indicates the inverse. Equation (6) is substituted in the corresponding second equation of (5), and the displacement vectors corresponding to the boundary DOFs are calculated as follows:

$$
\begin{aligned}
& \mathbf{u}_{b}^{(o)}=\left[\mathbf{K}_{b b}^{(o)}-\mathbf{K}_{b a}^{(o)} \mathbf{K}_{a a}^{(o)^{-1}} \mathbf{K}_{a b}^{(o)}\right]^{-1}\left[\mathbf{F}_{b}^{(o)}-\mathbf{K}_{b a}^{(o)} \mathbf{K}_{a a}^{(o)^{-1}} \mathbf{F}_{a}^{(o)}\right], \\
& \mathbf{u}_{b}^{(1)}=\left[\mathbf{K}_{b b}^{(1)}-\mathbf{K}_{b a}^{(1)} \mathbf{K}_{a a}^{(1)^{-1}} \mathbf{K}_{a b}^{(1)}\right]^{-1}\left[\mathbf{F}_{b}^{(1)}-\mathbf{K}_{b a}^{(1)} \mathbf{K}_{a a}^{(1)^{-1}} \mathbf{F}_{a}^{(1)}\right], \\
& \mathbf{u}_{b}^{(l)}=\left[\mathbf{K}_{b b}^{(l)}-\mathbf{K}_{b a}^{(l)} \mathbf{K}_{a a}^{(l)^{-1}} \mathbf{K}_{a b}^{(l)}\right]^{-1}\left[\mathbf{F}_{b}^{(l)}-\mathbf{K}_{b a}^{(l)} \mathbf{K}_{a a}^{(l)^{-1}} \mathbf{F}_{a}^{(l)}\right], \\
& \mathbf{u}_{b}^{(r)}=\left[\mathbf{K}_{b b}^{(r)}-\mathbf{K}_{b a}^{(r)} \mathbf{K}_{a a}^{(r)^{-1}} \mathbf{K}_{a b}^{(r)}\right]^{-1}\left[\mathbf{F}_{b}^{(r)}-\mathbf{K}_{b a}^{(r)} \mathbf{K}_{a a}^{(r)^{-1}} \mathbf{F}_{a}^{(r)}\right] .
\end{aligned}
$$

With respect to unstable substructures, it is not possible to derive the displacements in (6) and (7) because of the rank-deficient stiffness matrix. The resultant equilibrium equation at the interface DOFs is expressed as follows:

$$
\begin{aligned}
& \mathbf{K}_{b}^{(o)^{*}} \mathbf{u}_{b}^{(o)}=\mathbf{F}_{b}^{(o)^{*}}, \\
& \mathbf{K}_{b}^{(l)^{*}} \mathbf{u}_{b}^{(l)}=\mathbf{F}_{b}^{(l)^{*}}, \quad l=1,2, \ldots, r,
\end{aligned}
$$

where $\quad \mathbf{K}_{b}^{(o)^{*}}=\left[\mathbf{K}_{b b}^{(o)}-\mathbf{K}_{b a}^{(o)} \mathbf{K}_{a a}^{(o)^{-1}} \mathbf{K}_{a b}^{(o)}\right], \quad \mathbf{F}_{b}^{(o)^{*}}=\left[\mathbf{F}_{b}^{(o)}-\right.$ $\left.\mathbf{K}_{b a}^{(o)} \mathbf{K}_{a a}^{(o)^{-1}} \mathbf{F}_{a}^{(o)}\right], \quad \mathbf{K}_{b}^{(l)^{*}}=\left[\mathbf{K}_{b b}^{(l)}-\mathbf{K}_{b a}^{(l)} \mathbf{K}_{a a}^{(l)^{-1}} \mathbf{K}_{a b}^{(l)}\right]$, and $\mathbf{K}_{b}^{(l)^{*}}=$ $\left.\left[\mathbf{K}_{b b}^{(l)}-\mathbf{K}_{b a}^{(l)} \mathbf{K}_{a a}^{(l)}\right)^{-1} \mathbf{K}_{a b}^{(l)}\right], l=1,2, \ldots, r$.

Furthermore, $m$ compatibility conditions are assumed at the interface DOFs, and thus the coefficient matrix $\mathbf{A}$ at (2) that represents the compatibility condition corresponds to a $m \times 2 m$ matrix. Specifically, $m$ corresponds to the number of common DOFs at the interfaces. The equilibrium equations of (8) and the compatibility conditions of (2) are used with respect to (3) to obtain an equilibrium equation constrained by the adjacent substructures. The resulting displacements are expressed by a $2 m \times 1$ displacement vector $\mathbf{u}$. The actual displacements at the boundary DOFs are expressed by a $m \times 1$ vector due to the compatibility conditions such that the displacements at interface nodes are the same. The displacements at the internal DOFs are estimated by using (6) that is derived by the Guyan condensation method.

Example 1 (addition of supports). A planar truss structure in Figure 2 is modeled by 30 bar elements and 14 nodes by using a finite element model. The cross-sectional area, material density, and elasticity modulus correspond to $0.0025 \mathrm{~m}^{2}$, $7,860 \mathrm{~kg} / \mathrm{m}^{3}$, and $200 \mathrm{GPa}$, respectively. The truss structure is subjected to $50 \mathrm{~N}$ in the downward direction at node 5 . The first case involves the reanalysis of the modified structure due to an installation of a roller support at node 4 as shown in Figure 3.

The equilibrium equation of the original truss structure is expressed as follows: 


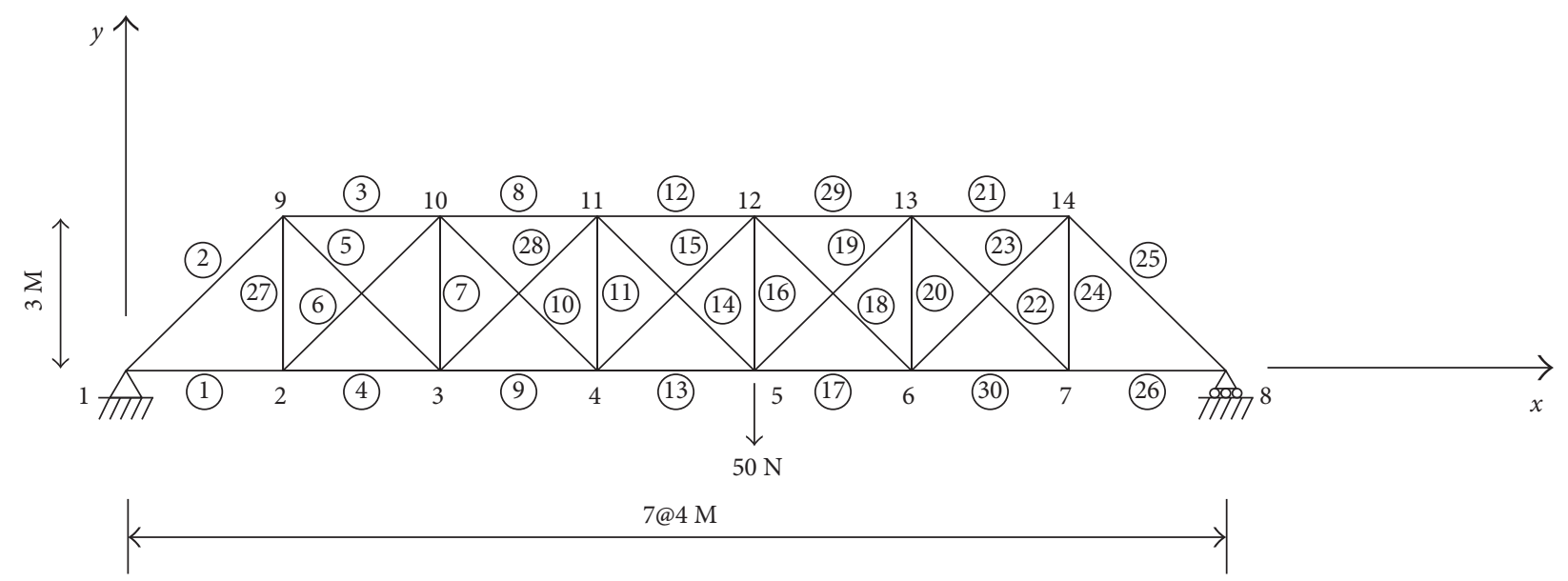

FIGURE 2: Original truss structure.

$$
\mathbf{K u}=\mathbf{F},
$$

where $\mathbf{K}$ denotes a $25 \times 25$ stiffness matrix and $\mathbf{u}$ and $\mathbf{F}$ represent $25 \times 1$ displacement and force vectors, respectively. Based on the Guyan condensation method, the equilibrium equation corresponding to the interface DOFs at node 4 is expressed as follows:

$$
\mathbf{K}_{b} \mathbf{u}_{b}=\mathbf{F}_{b},
$$

where $\mathbf{u}_{b}=\left[\begin{array}{ll}u_{4} & v_{4}\end{array}\right]^{T}$ and $u$ and $v$ represent the horizontal and vertical displacements, respectively.

The constraint condition to restrict the vertical displacement at node 4 is expressed as follows:

$$
\mathbf{A} \mathbf{u}_{b}=\mathbf{0}
$$

where $\mathbf{A}=\left[\begin{array}{cc}u_{4} & v_{4} \\ 0 & 1\end{array}\right]$. Equations (10) and (11) are used with respect to the proposed method in (3) to calculate the displacements due to the support coupling. Additionally, the displacements corresponding to the DOFs of the internal nodes are calculated by using the remaining equilibrium equations obtained by the Guyan condensation method.

2.2.2. Addition of Floating Substructures. This section considers the reanalysis of a modified structure due to the addition of rank-deficient floating substructures. The condensation method and the generalized inverse method assume a positive-definite full-rank stiffness matrix. The generalized inverse method is modified to obtain a constrained response due to the rank-deficient stiffness matrix.

It is assumed that the initial structure B in Figure 4(b) is modified by attaching a substructure A that is composed of five elements connected at node $I$ as shown in Figure 4(a). The substructure corresponds to an unstable structure in contrast to the previous case. The equilibrium equations of $h$ DOFs at $(n-1)$ nodes of the initial truss structure in Figure 4(b) are expressed as follows:

$$
\mathbf{K}_{o} \mathbf{u}_{o}=\mathbf{F}_{o},
$$

where $\mathbf{K}_{o}, \mathbf{u}_{o}$, and $\mathbf{F}_{o}$ represent the $h \times h$ stiffness matrix, $h \times 1$ displacement vector, and $h \times 1$ force vector, respectively, which is expressed as follows:

$$
\begin{aligned}
\mathbf{K}_{o} & =\left[\begin{array}{cccccccc}
k_{11} & \cdots & k_{1(I-1)} & k_{1(I+1)} & \cdots & k_{1(I+5)} & \cdots & k_{1 h} \\
\vdots & \ddots & \vdots & \vdots & \ddots & \vdots & \ddots & \vdots \\
k_{(I-1) 1} & \cdots & k_{(I-1)(I-1)} & k_{(I-1)(I+1)} & \cdots & k_{(I-1)(I+5)} & \cdots & k_{(I-1) h} \\
k_{(I+1) 1} & \cdots & k_{(I+1)(I-1)} & k_{(I+1)(I+1)} & \cdots & k_{(I+1)(I+5)} & \cdots & k_{(I+1) h} \\
\vdots & \ddots & \vdots & \vdots & \ddots & \vdots & \ddots & \vdots \\
k_{(I+5) 1} & \cdots & k_{(I+5)(I-1)} & k_{(I+5)(I+1)} & \cdots & k_{(I+5)(I+5)} & \cdots & k_{(I+5) h} \\
\vdots & \ddots & \vdots & \vdots & \ddots & \vdots & \ddots & \vdots \\
k_{h 1} & \cdots & k_{h(I-1)} & k_{h(I+1)} & \cdots & k_{h(I+5)} & \cdots & k_{h h}
\end{array}\right], \\
\mathbf{u}_{o} & =\left[\begin{array}{c}
u_{1} \\
\vdots \\
u_{(I-1)} \\
u_{(I+1)} \\
\vdots \\
u_{(I+5)} \\
\vdots \\
u_{h}
\end{array}\right], \\
\mathbf{F}_{o} & =\left[\begin{array}{cccccc} 
\\
f_{1} \\
\vdots \\
f_{(I-1)} \\
f_{(I+1)} \\
\vdots \\
f_{(I+5)} \\
\vdots \\
f_{h}
\end{array}\right]
\end{aligned}
$$

The equations are divided into equations corresponding to the internal DOFs and interface DOFs as follows:

$$
\left[\begin{array}{ll}
\mathbf{K}_{a a}^{(o)} & \mathbf{K}_{a b}^{(o)} \\
\mathbf{K}_{b a}^{(o)} & \mathbf{K}_{b b}^{(o)}
\end{array}\right]\left[\begin{array}{c}
\mathbf{u}_{a}^{(o)} \\
\mathbf{u}_{b}^{(o)}
\end{array}\right]=\left[\begin{array}{c}
\mathbf{F}_{a}^{(o)} \\
\mathbf{F}_{b}^{(o)}
\end{array}\right] .
$$




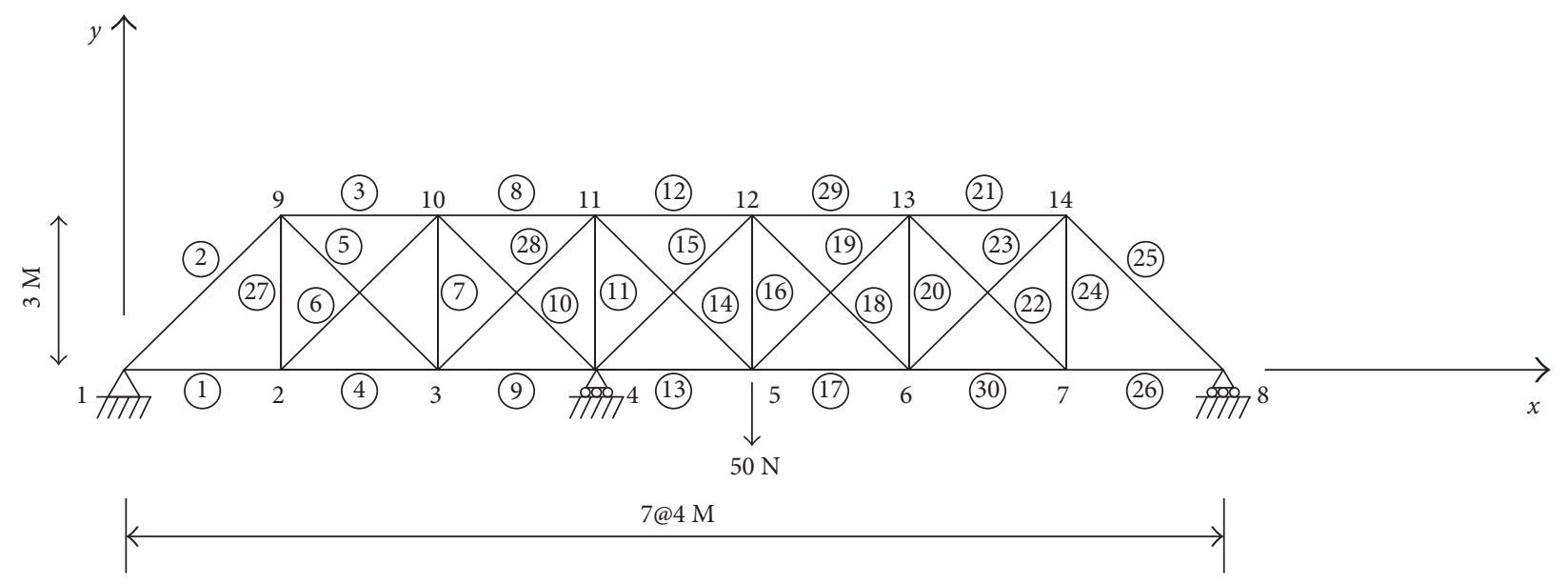

Figure 3: Modified truss structure adding a roller support.

The Guyan condensation method is used to derive the equilibrium equation at the boundary DOFs as follows:

$$
\mathbf{K}_{b}^{(o)^{*}} \mathbf{u}_{b}^{(o)}=\mathbf{F}_{b}^{(o)^{*}}
$$

where $\quad \mathbf{K}_{b}^{(o)^{*}}=\left[\mathbf{K}_{b b}^{(o)}-\mathbf{K}_{b a}^{(o)} \mathbf{K}_{a a}^{(o)^{-1}} \mathbf{K}_{a b}^{(o)}\right], \quad \mathbf{F}_{b}^{(o)^{*}}=\left[\mathbf{F}_{b}^{(o)}-\right.$ $\left.\mathbf{K}_{b a}^{(o)} \mathbf{K}_{a a}^{(o)^{-1}} \mathbf{F}_{a}^{(o)}\right]$, and $\mathbf{u}_{b}^{(o)}=\left[\begin{array}{lll}u_{I+1} & \cdots & u_{I+5}\end{array}\right]^{T}$. The added substructure in Figure 4(a) is composed of six nodes, and five of the nodes correspond to interface nodes. Its equilibrium equation is expressed as follows:

$$
\widehat{\mathbf{K}} \widehat{\mathbf{u}}=\widehat{\mathbf{F}} \text {, }
$$

where

$$
\begin{aligned}
\widehat{\mathbf{K}} & =\left[\begin{array}{cccc}
\widehat{k}_{11} & \widehat{k}_{12} & \cdots & \widehat{k}_{16} \\
\widehat{k}_{21} & \widehat{k}_{22}^{(I+1)} & \cdots & \widehat{k}_{26} \\
\vdots & \vdots & \ddots & \vdots \\
\widehat{k}_{61} & \widehat{k}_{62} & \cdots & \widehat{k}_{66}
\end{array}\right], \\
\widehat{\mathbf{u}} & =\left[\begin{array}{c}
\widehat{u}_{1} \\
\widehat{u}_{2} \\
\vdots \\
\widehat{u}_{6}
\end{array}\right], \\
\widehat{\mathbf{F}} & =\left[\begin{array}{c}
0 \\
0 \\
\vdots \\
0
\end{array}\right] .
\end{aligned}
$$

$\widehat{\mathbf{K}}$ denotes a rank-deficient floating stiffness matrix. Thus, the equilibrium equations in (15) and (16) are modified to obtain a full-rank stiffness matrix as follows:

$$
\overline{\mathbf{K}}^{*} \mathbf{u}^{*}=\mathbf{F}^{*}+\mathbf{C u}^{*},
$$

where $\overline{\mathbf{K}}^{*}=\left[\begin{array}{cc}\mathbf{K}_{b}^{(o)^{*}} & 0 \\ 0 & \widehat{\mathbf{K}}+\mathbf{I}\end{array}\right], \mathbf{u}^{*}=\left[\begin{array}{c}\mathbf{u}_{b}^{(o)} \\ \widehat{\mathbf{u}}\end{array}\right], \mathbf{F}^{*}=\left[\begin{array}{c}\mathbf{F}_{b}^{(o)^{*}} \\ \widehat{\mathbf{F}}\end{array}\right]$, and $\mathbf{C}=\left[\begin{array}{ll}0 & 0 \\ 0 & \mathbf{I}\end{array}\right]$

The compatibility conditions at the interface nodes are expressed as follows:

$$
\mathbf{A}^{*} \mathbf{u}^{*}=\mathbf{0} .
$$

Equations (18) and (19) are substituted in (3), and the result is arranged to calculate the modified equilibrium equation as follows:

$$
\mathbf{S u}^{*}=\mathbf{R F}^{*} \text {, }
$$

where $\quad \mathbf{S}=\left[\mathbf{I}-\overline{\mathbf{K}}^{*^{-1}} \mathbf{C}+\overline{\mathbf{K}}^{*^{-1 / 2}}\left(\mathbf{A}^{*} \overline{\mathbf{K}}^{*^{-1 / 2}}\right)^{+} \mathbf{A}^{*} \overline{\mathbf{K}}^{*^{-1}} \mathbf{C}\right]$ and $\mathbf{R}=\overline{\mathbf{K}}^{*^{-1}}-\overline{\mathbf{K}}^{*^{-1 / 2}}\left(\mathbf{A}^{*} \overline{\mathbf{K}}^{*^{-1 / 2}}\right)^{+} \mathbf{A}^{*} \overline{\mathbf{K}}^{*^{-1}}$.

Equation (20) represents the equilibrium equation of the modified structure that corresponds to the interface DOFs. The displacement responses corresponding to the internal DOFs are also estimated by using the first equation in (14).

Example 2. The example considers a reanalysis of the truss structure to attach a substructure composed of elements $a, b$, and $c$ at nodes 14,8 , and 7 , respectively, as shown in Figure 5. The equilibrium equations corresponding to nodes 7,8 , and 14 are obtained by the Guyan condensation method with respect to the initial truss structure. Additionally, the equilibrium equation of the floating substructure and the compatibility conditions between the initial structure and the substructure are obtained. The aforementioned equations are substituted in (20) to explicitly obtain displacements at the boundary DOFs of the modified structure. In the next step, the responses at the internal DOFs are predicted from the Guyan condensation method.

2.3. Removal of Substructures. The removal of substructures from a structure leads to a decrease in the initial DOFs. The removal indicates the release of compatibility conditions with respect to the interface nodes, and the structure is separated. A decoupling process is performed as a reversal 

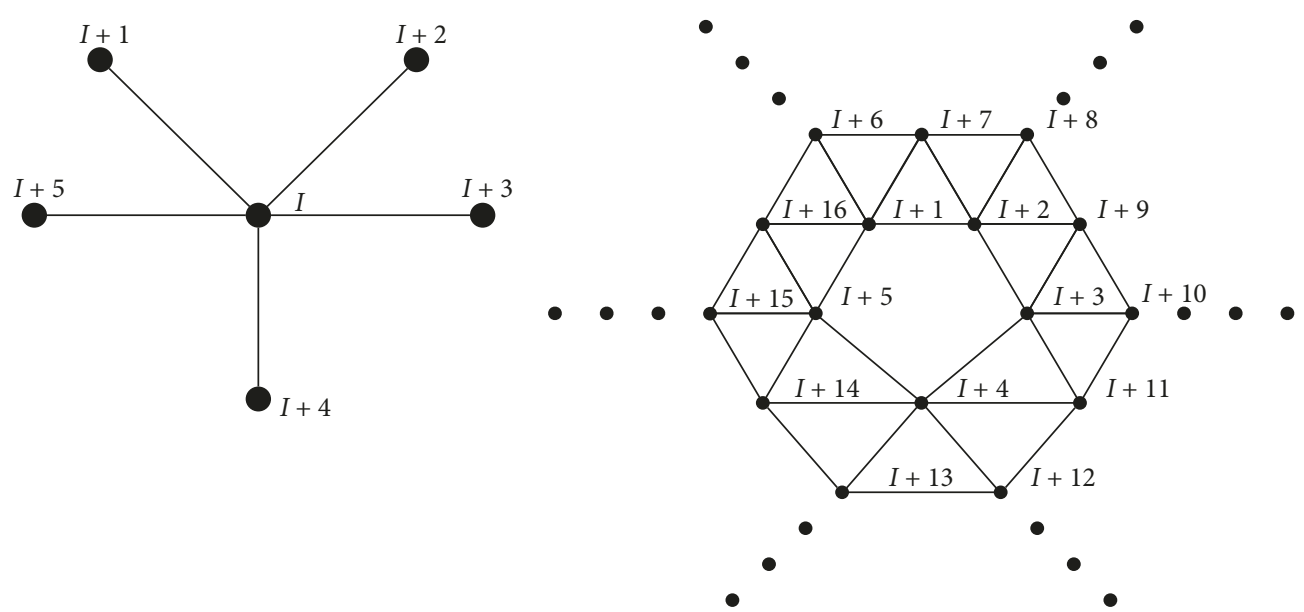

(a)

(b)

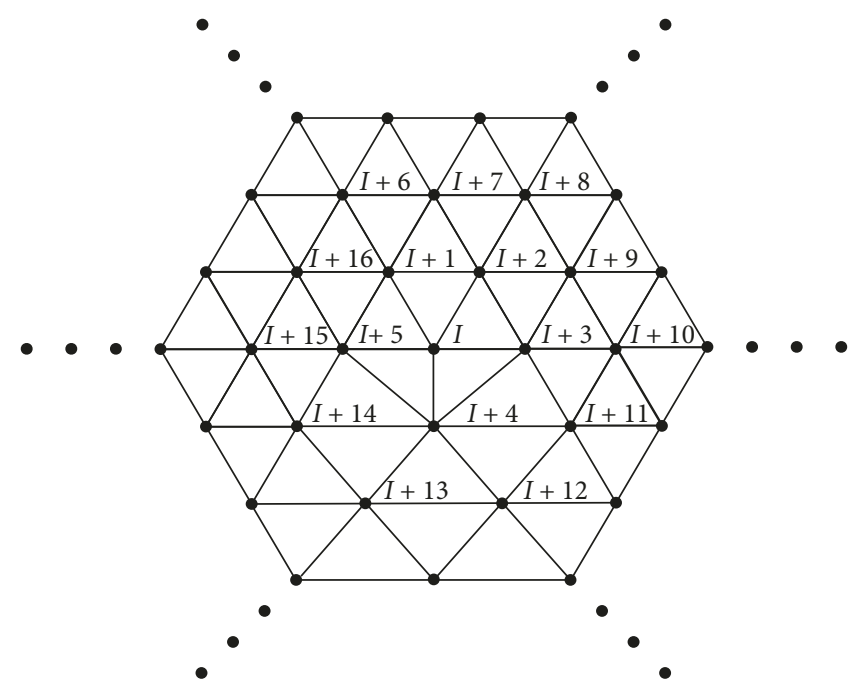

(c)

FIGURE 4: Coupling and decoupling of substructures: (a) substructure A; (b) initial structure B; (c) entire structure.

of the coupling process. The removal of five elements connected at node $I$ as shown in Figure 4(c) is considered. Substructure A including node $I$ in Figure 4(a) is excluded from the entire structure, and the remaining substructure $B$ is isolated as shown in Figure 4(b). The release of the DOFs at the boundary nodes $(I+1)-(I+5)$ affects the responses at the other end nodes $(I+6)-(I+16)$. The equilibrium equations corresponding to nodes $(I+1)-$ $(I+16)$ are considered as the boundary DOFs at removal in contrast to the coupling process because it is necessary to constrain the responses at nodes $(I+6)-(I+16)$ by the internal elements.

Substructure A is divided into an internal node and five boundary nodes, and the Guyan condensation method is applied to express this as follows:

$$
\begin{aligned}
{\left[\begin{array}{cc}
\mathbf{K}_{a a}^{(A)} & \mathbf{K}_{a b}^{A} \\
\mathbf{K}_{b a}^{(A)} & \mathbf{K}_{b b}^{(A)}
\end{array}\right]\left[\begin{array}{l}
\mathbf{u}_{a}^{(A)} \\
\mathbf{u}_{b}^{(A)}
\end{array}\right] } & =\left[\begin{array}{l}
\mathbf{F}_{a}^{(A)} \\
\mathbf{F}_{b}^{(A)}
\end{array}\right], \\
\mathbf{K}_{b}^{(A)^{*}} \mathbf{u}_{b}^{(A)} & =\mathbf{F}_{b}^{(A)^{*}},
\end{aligned}
$$

where

$$
\begin{aligned}
\mathbf{K}_{b}^{(A)^{*}} & =\left[\mathbf{K}_{b b}^{(A)}-\mathbf{K}_{b a}^{(A)} \mathbf{K}_{a a}^{(A)^{-1}} \mathbf{K}_{a b}^{(A)}\right]=\left[\begin{array}{cccc}
k_{11}^{(A)} & k_{12}^{(A)} & \cdots & k_{15}^{(A)} \\
k_{21}^{(A)} & k_{22}^{(A)} & \cdots & k_{25}^{(A)} \\
\vdots & \vdots & \ddots & \vdots \\
k_{51}^{(A)} & k_{52}^{(A)} & \cdots & k_{55}^{(A)}
\end{array}\right] \\
\widehat{\mathbf{u}}_{b}^{(A)} & =\left[\begin{array}{c}
\widehat{u}_{(I+1)}^{(A)} \\
\widehat{\boldsymbol{u}}_{(I+2)}^{(A)} \\
\vdots \\
\widehat{u}_{(I+5)}^{(A)}
\end{array}\right] \\
\mathbf{F}_{b}^{(A)^{*}} & =\left[\mathbf{F}_{b}^{(A)}-\mathbf{K}_{b a}^{(A)} \mathbf{K}_{a a}^{(A)^{-1}} \mathbf{F}_{a}^{(A)}\right]
\end{aligned}
$$

The equilibrium equations at the 16 boundary nodes of the structure $B$ are expressed as follows:

$$
\mathbf{K}_{b}^{(B)^{*}} \mathbf{u}_{b}^{(B)}=\mathbf{F}_{b}^{(B)^{*}},
$$




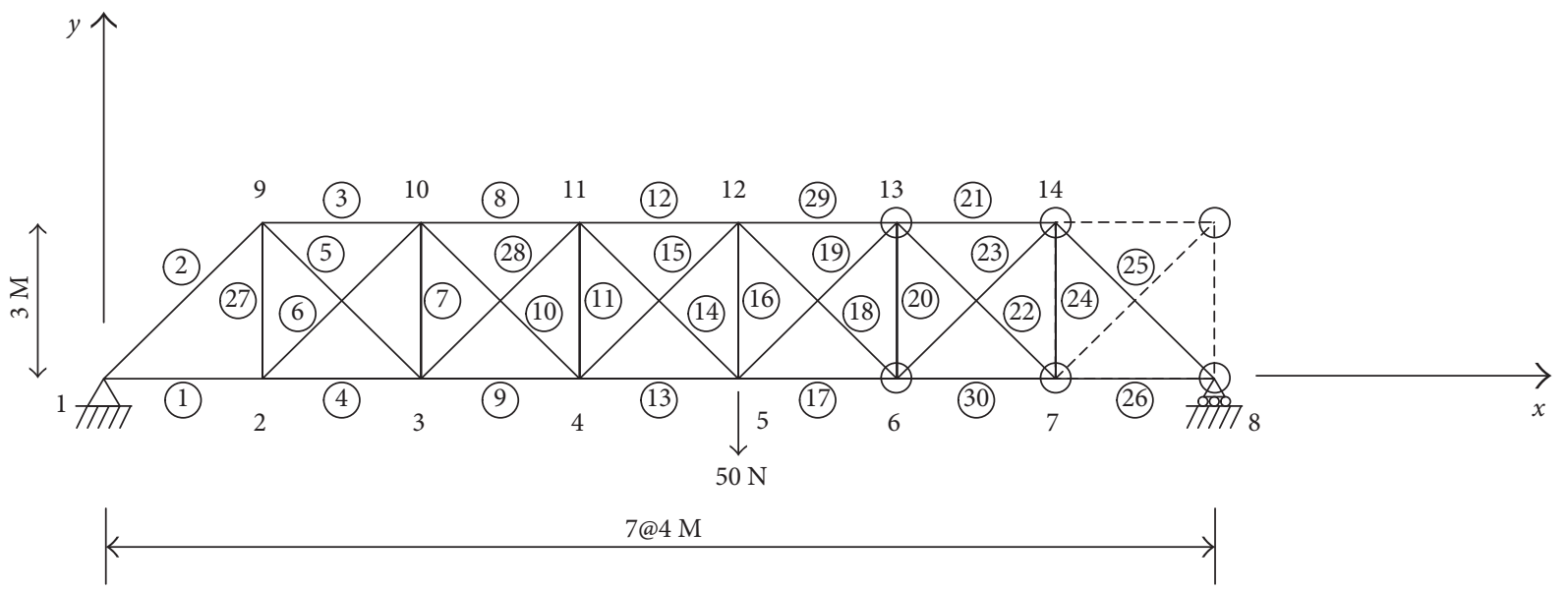

(a)

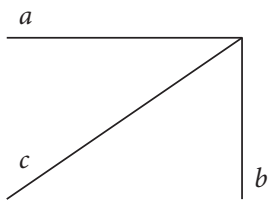

(b)

Figure 5: Coupling and decoupling of a substructure composed of three elements. (a) Initial and modified truss structure. The dashed line represents the added substructure. (b) Added floating substructure.

where

$$
\begin{aligned}
& \mathbf{K}_{b}^{(B)^{*}}=\left[\mathbf{K}_{b b}^{(B)}-\mathbf{K}_{b a}^{(B)} \mathbf{K}_{a a}^{(B)^{-1}} \mathbf{K}_{a b}^{(B)}\right]=\left[\begin{array}{cccc}
k_{1,1}^{(B)} & k_{1,2}^{(B)} & \cdots & k_{1,16}^{(B)} \\
k_{2,1}^{(B)} & k_{2,2}^{(B)} & \vdots & k_{2,16}^{(B)} \\
\vdots & \vdots & \ddots & \vdots \\
k_{16,1}^{(B)} & k_{16,2}^{(B)} & \cdots & k_{16,16}^{(B)}
\end{array}\right] \\
& \mathbf{u}_{b}^{(B)}=\left[\begin{array}{llll}
u_{(I+1)}^{(B)} & u_{(I+2)}^{(B)} & \cdots & u_{(I+16)}^{(B)}
\end{array}\right] \\
& \mathbf{F}_{b}^{(B)^{*}}=\left[\mathbf{F}_{b}^{(B)}-\mathbf{K}_{b a}^{(B)} \mathbf{K}_{a a}^{(B)^{-1}} \mathbf{F}_{a}^{(B)}\right]
\end{aligned}
$$

The constraints of compatibility conditions at free interface DOFs are established at the interfaces and expressed as follows:

$$
\mathbf{A u}=\mathbf{0},
$$

where $\mathbf{u}=\left[\begin{array}{ll}\mathbf{u}_{b}^{(A) T} & \mathbf{u}_{b}^{(B) T}\end{array}\right]^{T}$ and

$$
\mathbf{A}=\left[\begin{array}{ccccccccccc}
u_{1}^{(A)} & u_{2}^{(A)} & u_{3}^{(A)} & u_{4}^{(A)} & u_{5}^{(A)} & & \widehat{u}_{1}^{(B)} & \widehat{u}_{2}^{(B)} & \widehat{u}_{3}^{(B)} & \widehat{u}_{4}^{(B)} & \widehat{u}_{5}^{(B)} \\
1 & 2 & 3 & 4 & 5 & \cdots & -1 & 0 & 0 & 0 & 0 \\
0 & 1 & 0 & 0 & 0 & \cdots & 0 & -1 & 0 & 0 & 0 \\
0 & 0 & 1 & 0 & 0 & \cdots & 0 & 0 & -1 & 0 & 0 \\
0 & 0 & 0 & 1 & 0 & \cdots & 0 & 0 & 0 & -1 & 0 \\
0 & 0 & 0 & 0 & 1 & \cdots & 0 & 0 & 0 & 0 & -1
\end{array}\right]_{5 \times 21}
$$

Equations (22), (24), and (26) are substituted in (20) to derive the constrained equilibrium equation as follows:

$$
\mathbf{K}^{*} \mathbf{u}=\mathbf{F}^{*} \text {. }
$$

Equation (28) describes the synthesized system.

The decoupling indicates the release of DOFs at the interface nodes. The boundary DOFs of the substructure B include the DOFs at nodes $(I+6)-(I+16)$ and the DOFs at interface nodes $(I+1)-(I+5)$ to satisfy the compatibility conditions. The displacements after removal are predicted by solving simultaneous equations composed of the constrained equilibrium equations that correspond to the DOFs of nodes $(I+6)-(I+16)$ in (28) and the unconstrained equilibrium equations at the DOFs of the nodes $(I+1)-(I+5)$ in $(22)$. The displacements due to the removal of substructures are explicitly obtained by solving the simultaneous equations of the unconstrained equilibrium equations at the interface DOFs and the constrained equilibrium equations at the DOFs of the adjacent nodes.

Example 3. The reanalysis due to the support removal at node 4 of the truss structure in Figure 3 is considered. The removal of the support affects the displacements of all the DOFs. The vertical displacement is described by an unconstrained equilibrium equation because the constraint condition is released. The displacements corresponding to a DOF of the horizontal displacement at the support node and ten DOFs at the other nodes of the five elements (9), (10), (11), (15), and (13) are described by the constrained equilibrium equations. Thus, the displacements including the vertical displacement 
are predicted by solving the twelve simultaneous equations of unconstrained and constrained equations.

Example 4. The example considers the reanalysis of the remaining structure after removing the three elements $a, b$, and $c$ that are attached at node (15) in Figure 5(a). Seven elements corresponding to $21,22,23,24,25,26$, and 30 share the removed elements at three nodes, namely, 7, 8, and 14 . The equilibrium equations corresponding to the four released DOFs at nodes 7 and 14 and an equilibrium equation corresponding to the horizontal displacement released at node 8 are predicted by unconstrained equilibrium equations due to the release of the constraint conditions. The displacements corresponding to nodes 6 and 13 of the elements that share the interface nodes are constrained by the remaining structure. They are described by constrained equilibrium equations. The resulting responses are predicted by solving simultaneous equations of unconstrained and constrained equilibrium equations.

2.4. Change of Variables. The section considers the reanalysis of the modified structure due to changes of design variables. The number of DOFs is unchanged in this case. The approach commences with the assumption that the original structure is additionally constrained by elements of changed design variables. The changed elements are reinforced because of additional constraints.

A structure is divided into substructures composed of $s$ elements of changed design variables (stiffnesses) and other substructures, and thus equilibrium equations of the DOFs corresponding to the changed design variables are expressed by employing the Guyan method as follows:

$$
\begin{aligned}
& \mathbf{K}_{a}^{*} \mathbf{u}_{a}=\mathbf{F}_{a}^{*}, \\
& \mathbf{K}_{b}^{*} \mathbf{u}_{b}=\mathbf{F}_{b}^{*},
\end{aligned}
$$

where $\mathbf{K}_{b}^{*}=\left[\mathbf{K}_{b b}-\mathbf{K}_{b a} \mathbf{K}_{a a}^{-1} \mathbf{K}_{a b}\right], \mathbf{F}_{b}^{*}=\mathbf{F}_{b}-\mathbf{K}_{b a} \mathbf{K}_{a a}^{-1} \mathbf{F}_{a}$, and the subscripts $a$ and $b$ denote the internal and boundary elements, respectively. The equilibrium equations corresponding to the changed elements such that it is an unstable substructure are expressed as follows:

$$
\mathbf{K}_{c} \mathbf{u}_{c}=\mathbf{F}_{c}
$$

where the subscript $c$ denotes the elements of changed design variables. The compatibility conditions between two substructures are expressed as follows:

$$
\mathbf{A u}=\mathbf{0} .
$$

Equations (30)-(32) are inserted into (19) to obtain the resulting equation. However, the attachment of the element leads to an overestimated local stiffness in contrast to actual design variables. Accordingly, the ultimate displacement is adjusted as the proportion of the actual design stiffness values with respect to the combined stiffness values.

Example 5. This example involves estimating the changed displacements due to a change in the cross section of local elements in the design. The reanalysis of the structure is changed from the initial cross section of $0.0025 \mathrm{~m}^{2}$ of the element 20 to the cross section of $0.002 \mathrm{~m}^{2}$. The reduced cross-sectional area or the stiffness of the element 20 is used to correct the actual displacement response to explain the change in the cross section.

In this case, it is assumed that the element is additionally constrained by an element of the cross section corresponding to $0.0015 \mathrm{~m}^{2}$, and thus the resulting displacement corresponds to half of the constrained displacement because the changed cross section of $0.002 \mathrm{~m}^{2}$ corresponds to half the sum of the initial cross section of $0.0025 \mathrm{~m}^{2}$ and the additional cross section of $0.0015 \mathrm{~m}^{2}$. The equilibrium equations of the added elements, the corresponding elements of the initial structure, and the compatibility conditions at the ends of the corresponding elements are substituted in (20), and thus the actual displacements are obtained by the proportion of the design stiffness with respect to the sum of the initial stiffness and the added stiffness. The example exhibits that the reanalysis is explicitly performed.

\section{Conclusions}

The study provides exact methods of structural reanalysis given the addition and removal of substructures and changes in design variables. The method adopts a constraint concept and commences with a generalized inverse method to describe constrained displacements and the Guyan condensation method. The generalized inverse method is modified to describe the coupling of substructures and the decoupling of substructures from an entire structure based on a stable or unstable substructure. The validity of the proposed method is illustrated via five examples to examine the reanalysis of a truss structure.

\section{Conflicts of Interest}

The authors declare that there are no conflicts of interest regarding the publication of this paper.

\section{Acknowledgments}

This research was supported by the Chung-Ang University research grant in 2017. This research is supported by Basic Science Research Program through the National Research Foundation of Korea (NRF) funded by the Ministry of Education (NRF-2016R1D1A1A09918011).

\section{References}

[1] J. Sherman and W. J. Morrison, "Adjustment of an inverse matrix corresponding to changes in the elements of a given column or a given row of the original matrix," Annals of Mathematical Statistics, vol. 20, p. 621, 1949.

[2] M. Woodbury, Inverting Modified Matrices, Memorandum Report 42, Statistical Research Group, Princeton University, Princeton, NJ, USA, 1950.

[3] P. B. Nair, "Approximate static and dynamic reanalysis techniques for structural optimization," in Proceedings of the 3rd International Conference on Modern Practice in Stress and Vibration Analysis, MPSVA, pp. 295-302, Dublin, Ireland, September 1997. 
[4] W. W. Hager, "Updating the inverse of a matrix," SIAM Review, vol. 31, no. 2, pp. 221-239, 1989.

[5] M. A. Akgun, J. H. Garcelon, and R. T. Haftka, "Fast exact linear and non-linear structural reanalysis and the ShermanMorrison-Woodbury formulas," International Journal for Numerical Methods in Engineering, vol. 50, no. 7, pp. 15871606, 2001.

[6] U. Kirsch and S. Liu, "Exact structural reanalysis by a firstorder reduced basis approach," Structural Optimization, vol. 10, no. 3-4, pp. 153-158, 1995.

[7] U. Kirsh and S. H. Liu, "Structural reanalysis for general layout modifications," AIAA Journal, vol. 35, no. 2, pp. 382-388, 1997.

[8] C. Huang and G. Verchery, "An exact structural static reanalysis method," International Journal for Numerical Methods in Biomedical Engineering, vol. 13, no. 2, pp. 103-112, 1997.

[9] F. Cheng, H. Xiu, T. Xu, and H. Zhang, "The extrapolation method for structural static reanalysis of topological modifications with added degrees of freedom," in Proceedings of the International Conference on Consumer Electronics, Communications and Networks (CECNet), Xianning, China, March 2011.

[10] H. F. Liu, B. S. Wu, and Z. G. Li, "An efficient approach to structural static reanalysis with added support constraints," Structural Engineering and Mechanics, vol. 43, no. 3, pp. 273-285, 2012.

[11] H. F. Liu, B. S. Wu, Z. G. Li, and S. P. Zheng, "Structural static reanalysis for modification of supports," Structural and Multidisciplinary Optimization, vol. 50, no. 3, pp. 425-435, 2014.

[12] H. Liu and S. Yue, "An efficient method to structural static reanalysis with deleting support constraints," Structural Engineering and Mechanics, vol. 52, no. 6, pp. 1121-1134, 2014.

[13] P. Kolakowski, M. Wiklo, and J. Holnicki-Szule, "The virtual distortion method-a versatile reanalysis tool for structures and systems," Structural and Multidisciplinary Optimization, vol. 36, no. 3, pp. 217-234, 2008.

[14] Y. Liu, J. Ma, J. Nie, and S. Zhang, "Virtual distortion methodbased finite element model updating of bridges by using static deformation," Journal of Engineering Mechanics, vol. 143, no. 3, 2017.

[15] J. Garcia de Jalon and F. Viadero, "A new direct method for the simple and efficient reanalysis of structures," Computers \& Structures, vol. 21, no. 5, pp. 1059-1066, 1985.

[16] M. Cheikh and P. Coorevits, "Structural analysis with stiffness modification," in Proceedings of the European Congress on Computational Methods in Applied Sciences and Engineering (ECCOMAS 2000), Barcelona, Spain, September 2000.

[17] E. T. Lee and H. C. Eun, "Structural reanalysis for a modified structure," Canadian Journal of Civil Engineering, vol. 35, no. 9, pp. 1018-1023, 2008. 


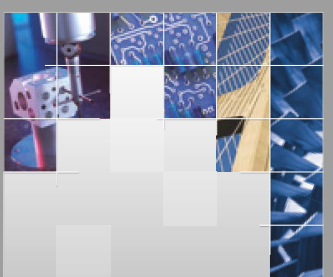

\section{Enfincering}
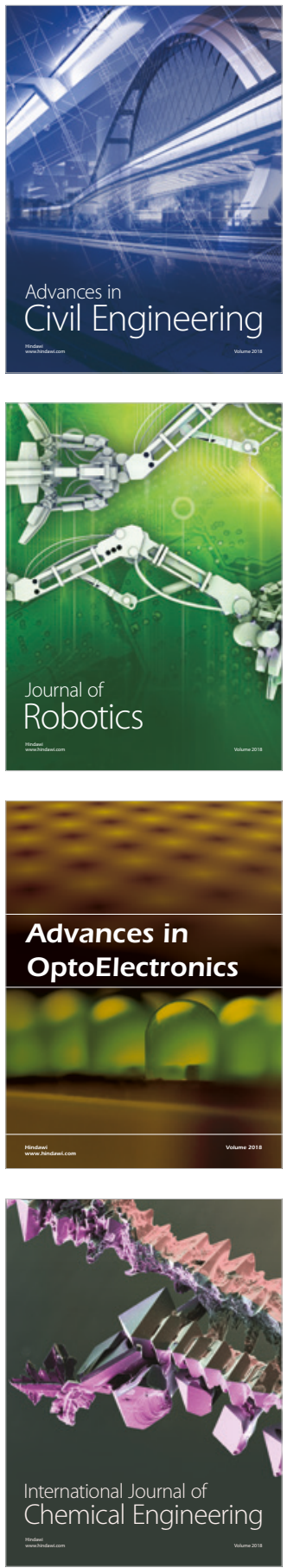

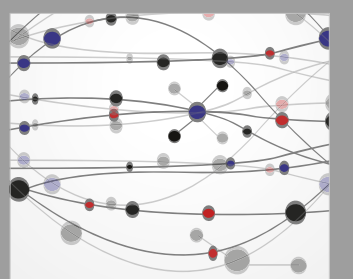

\section{Rotating \\ Machinery}

The Scientific World Journal

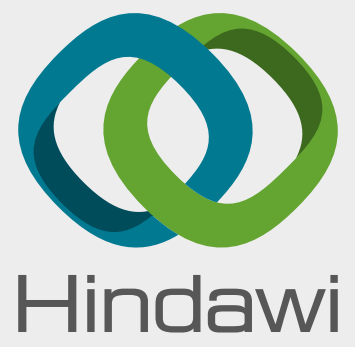

Submit your manuscripts at

www.hindawi.com
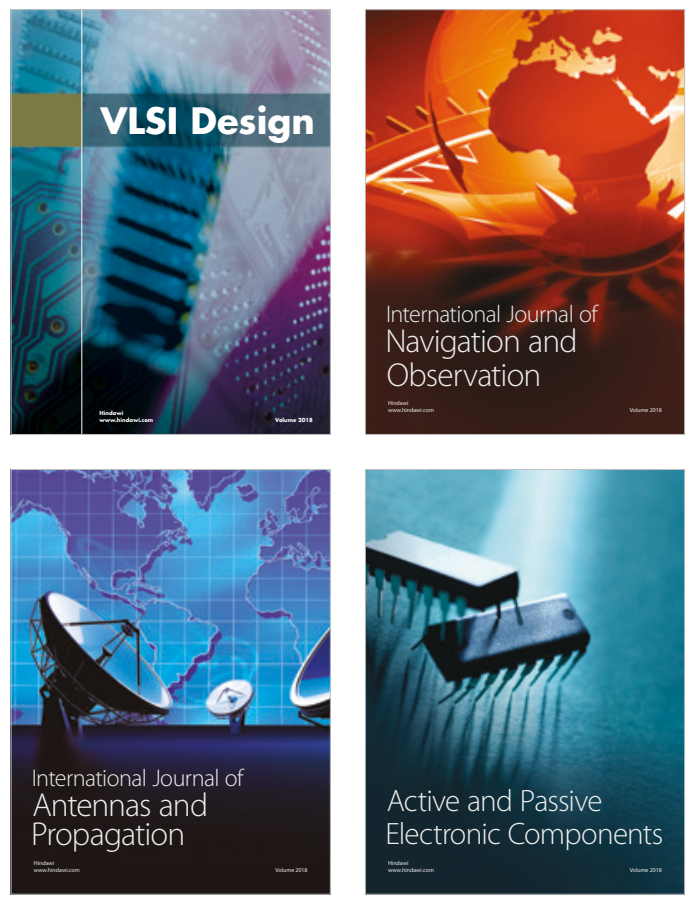
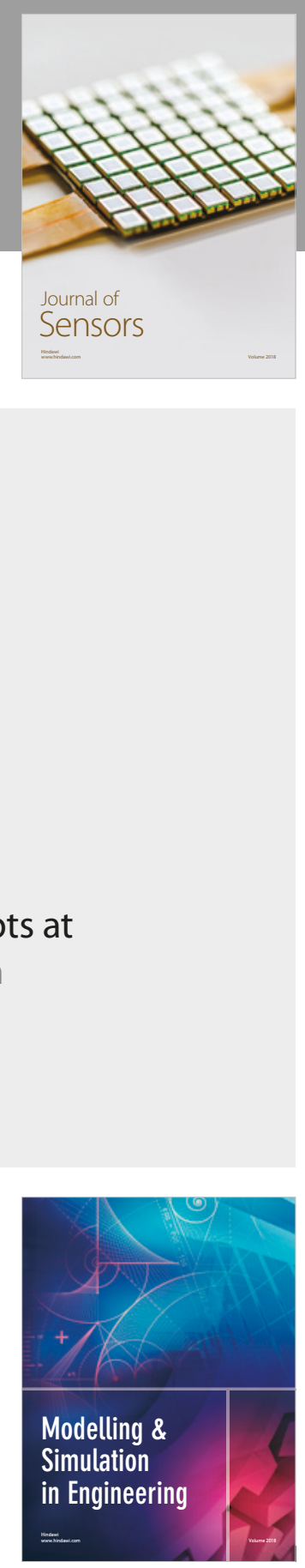

\section{Advances \\ Multimedia}
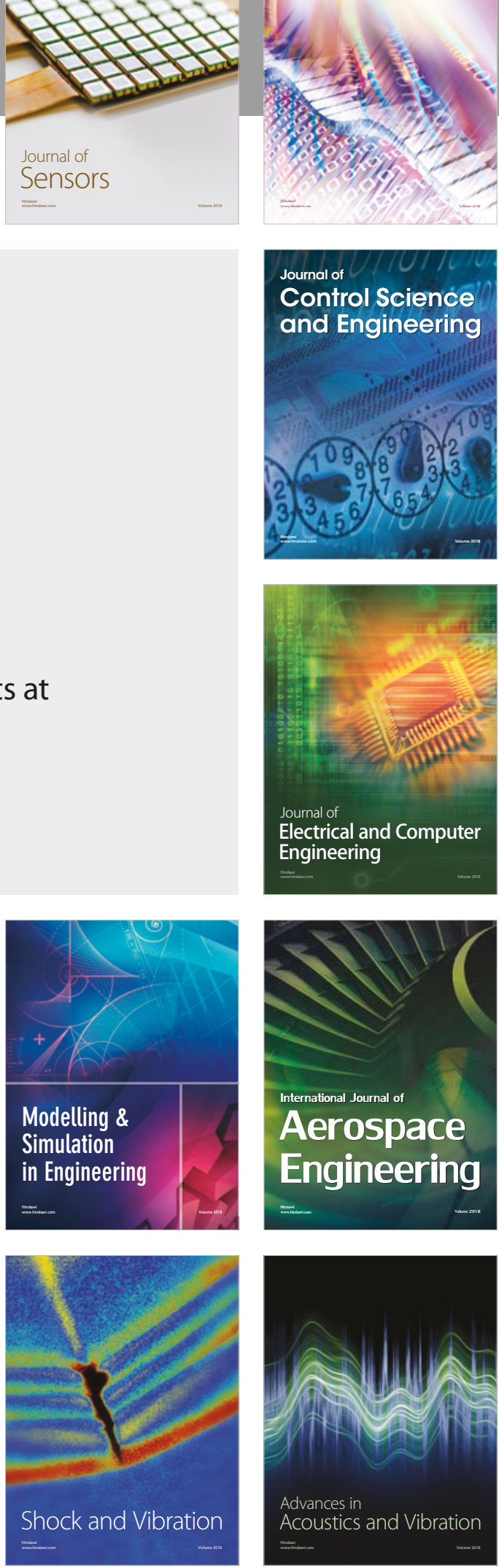